\title{
A Multi-Disciplinary Approach of a Patient with Extensive Arterio-Venous Malformations Undergoing a C-Section
}

\author{
Jing Song ${ }^{*}$, Sudheera Kokkada Sathyanarayana ${ }^{\#}$, Louvonia Boone, Vilma Joseph \\ Department of Anesthesiology, Montefiore Medical Center, Albert Einstein School of Medicine, New York, USA. \\ Email: *songjing6@yahoo.com
}

Received March $30^{\text {th }}, 2013$; revised April 30 $0^{\text {th }}, 2012$; accepted May $17^{\text {th }}, 2013$

Copyright (C) 2013 Jing Song et al. This is an open access article distributed under the Creative Commons Attribution License, which permits unrestricted use, distribution, and reproduction in any medium, provided the original work is properly cited.

\begin{abstract}
The presence of both uterine and arteriovenous malformation in a patient is rare. For these patients a cesarean section can be life threatening due to uncontrolled massive hemorrhage. Prevention and control of massive blood loss utilizing a multidisciplinary approach can be lifesaving. We present a case report of a 33 years old pregnant woman at 35 weeks of gestation diagnosed with an extensive uterine arteriovenous malformation and innumerable tortuous vessels who was scheduled for a cesarean section. Her previous vaginal delivery was complicated by significant bleeding requiring uterine artery embolization. Pre-operative prophylactic aortic and right iliac artery balloon occlusion catheters were placed under monitored anesthesia care. The extracorporeal life support team was available to initiate veno-venous or arteriovenous bypass. Cesarean section was performed with careful identification of the uterine vessels under ultrasound guidance and ultimately the bleeding was well controlled. Postoperatively, the patient underwent uterine artery embolization. It is our strong belief that although we did not face a major disaster during this cesarean section, our comprehensive plan and multi-disciplinary approach were essential to ensuring the safety of the parturient and newborn.
\end{abstract}

Keywords: Uterine Arteriovenous Malformation; Pelvic Arteriovenous Malformation; Cesarean Section; Management Anesthesia Care; Extracorporeal Life Support

\section{Introduction}

A pelvic arteriovenous malformation (AVM) is characterized by an abnormal communication between arteries and veins with localized proliferation, bypassing the capillary system. A severe pelvic AVM extending to the uterus and ovaries is a rare condition. The actual incidence of uterine and pelvic AVM is unknown [1-3]. It is usually more common in women than in men [1]. There are two types of uterine and pelvic AVM: congenital and acquired. A congenital AVM is the result of an abnormal embryologic development. An acquired AVM in women is most often related to previous gynecologic or pelvic surgery, pelvic trauma, molar disease, choriocarcinoma or childbirth [4].

Performing a cesarean section in a patient with uterine and pelvic AVM would be a major challenge to obstetricians and anesthesiologists, due to the risk of a massive hemorrhage. We present a case of a patient with an extensive pelvic and uterine AVM requiring a cesarean

\footnotetext{
${ }^{*}$ Corresponding author.
}

${ }^{\#}$ Co-first author. section (C-section). The focus of this case report is the construction of a feasible plan to manage a potentially catastrophic clinical scenario.

\section{Case Report}

A 33 year-old multigravida female at 35 weeks of gestation was admitted for an elective c-section. Her past medical history was significant for a molar pregnancy, pelvic and uterine AVM, multiple dilation and curettages, and two episodes of uterine artery embolization for recurrent vaginal bleeding after spontaneous vaginal deliveries. Pre-operative pelvic angiogram showed a large uterine AVM with innumerable tortuous vessels within the pelvis (Figure 1) Doppler ultrasound of the uterus revealed extensive vasculature of the anterior cervix extending laterally and posteriorly (Figure 2). There was an area with relatively fewer large vessels in the inferior-anterior aspect of the uterus body, which was anterior to the fetal head (Figure 3). Pre-operatively, several multidisciplinary meetings were conducted with representatives from obstetrics, anesthesiology, interventional radi- 
ology, vascular surgery, cardiothoracic surgery, perfusion medicine, and neonatology. A general anesthetic was planned for the $\mathrm{C}$-section and fetal well-being was assessed by ultrasound before surgery.

On arrival to the operating room, the patient's blood pressure was $123 / 69 \mathrm{mmHg}$; heart rate was 87 beats $/ \mathrm{min}$ with a hemoglobin of $11.5 \mathrm{~g} / \mathrm{dL}$. Two large-bore peripheral venous catheters, a femoral venous central line $(8.5$ F cordis) and a femoral arterial line were established. The patient was hydrated. In anticipation of massive hemorrhage, a prophylactic arterial occlusion balloon

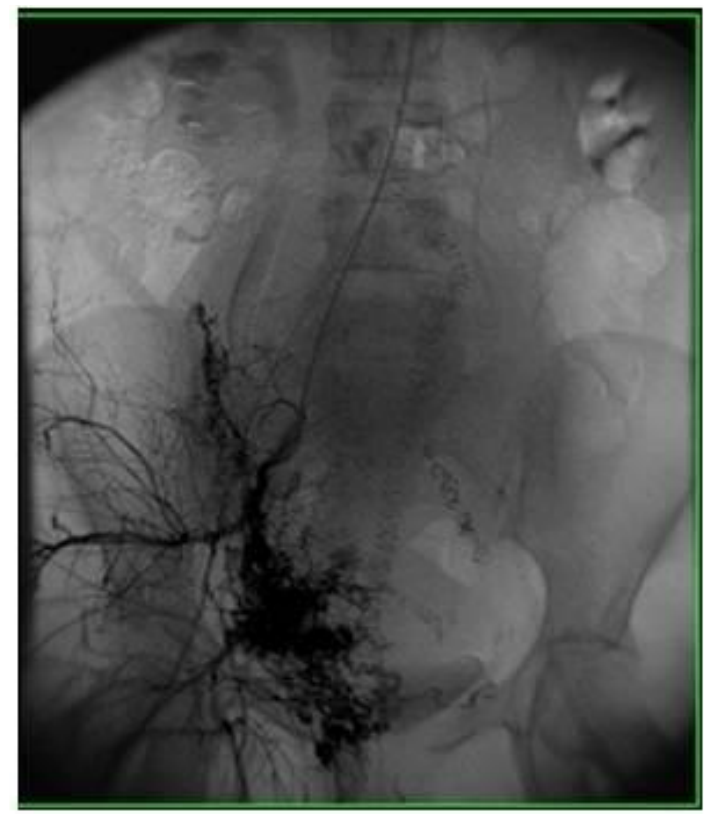

Figure 1. Pelvic angiogram showing arteriovenous malformation in the pelvis with extensive collateral arterial supply.

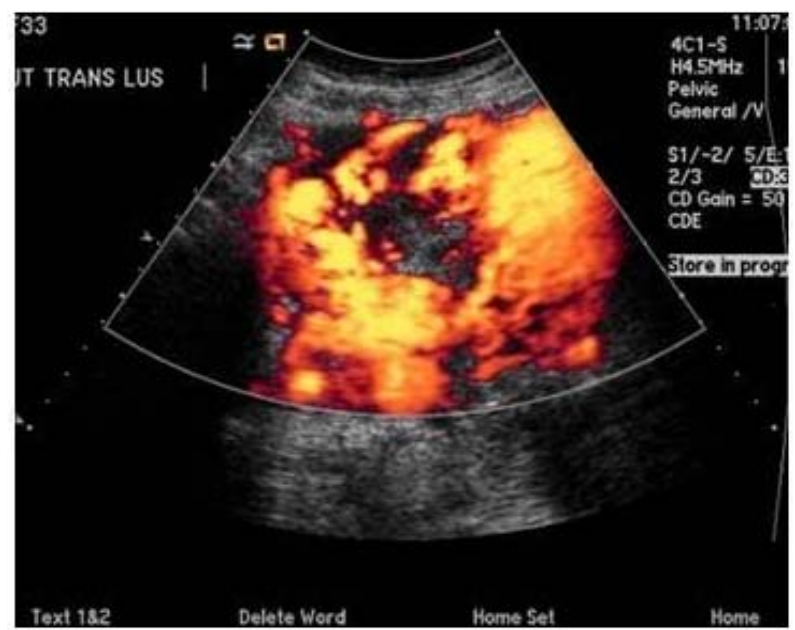

Figure 2. Transvaginal Doppler ultrasound showing extesive vascularity in the periphery of the uterus, involving virtually the entire anterior uterus.

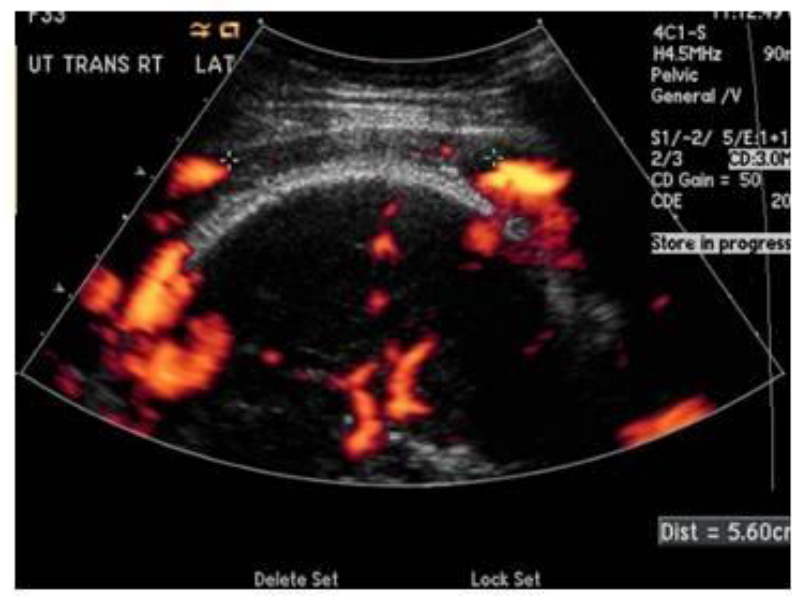

Figure 3. Transvaginal Doppler showing relative area with out large vessels in the inferior anterior uterus anterior to the fetal head.

catheter was placed into the distal aorta by the radiologist. The patient received mild sedation with $2 \mathrm{mg}$ of midazolam and $100 \mathrm{mcg}$ of fentanyl during the approximately one hour procedure. Then following pre oxygenation, the patient was induced. Intubation was achieved with a rapid sequence induction of $200 \mathrm{mg}$ of propofol and 100 $\mathrm{mg}$ of succinylcholine. The endotracheal tube was secured and general anesthesia was initiated.

Prior to incision for the C-section, a color doppler ultrasound of the uterus was used by the obstetrician. To minimize the risk of massive bleeding, a careful identifycation of normal as well as abnormal vessels in the area of the AVM was attempted. Blood and blood products including fresh frozen plasma and platelets were readily available. A fast flow fluid warmer for rapid transfusion was set up as well. The extracorporeal life support (ECLS) team was on standby for a possible immediate veno-venous or arteriovenous bypass. After a half hour search with the color doppler ultrasound, a half $\mathrm{cm}$ in diameter area with relatively few large vessels was localized. The dissection of the uterus was prolonged, but eventually the baby was delivered. The concentration of volatile agents was subsequently reduced and oxytocin was administered to minimize the risk of uterine atony and hemorrhage. Anesthesia was maintained with a low to moderate concentration of sevoflurane with $50 \%$ concentration of oxygen, $300 \mathrm{mcg}$ of fentanyl, and $30 \mathrm{mg}$ of rocuronium for the remaining period of the $\mathrm{C}$-section and the bilateral tubal ligation.

A baby girl with APGAR scores of two at one minute and seven at five minutes was transferred to the NICU for further management. During the surgery, the aortic occlusion balloon remained deflated. No significant hemodynamic changes were noted. The patient received $5800 \mathrm{ml}$ of crystalloid and two units of packed red blood 
cells with an estimated blood loss of $2000 \mathrm{ml}$. Finally, the patient was transferred to interventional radiology suite for embolization of the uterine artery and was successfully extubated at the end of the procedure.

\section{Discussion}

An extensive and progressing uterine and pelvic AVM diagnosed in the antepartum period presents a significant risk for major uterine bleeding. AVMs have been known to be one of the most therapeutically difficult to manage once a life threatening hemorrhage has occurred. The key to management of AVM is appropriate preoperative management.

The safety of both parturient and fetus was our primary concern. To fulfill this goal, a multidisciplinary approach including obstetrics \& gynecology, anesthesiaology, invasive radiology, neonatal ICU, vascular surgery, cardiothoracic surgery and the perfusion service was conducted. A remarkable comprehensive surgical and anesthesia plan was established. It was recognized that the critical points to ensure an effective and successful operation included 1) a consolidated and practical surgerical and anesthetic plan; 2) good communication and coordination amongst all related services; 3 ) immediate availability of blood/blood products and all advanced resuscitation equipment including an ECLS.

Uterine and pelvic AVM has been classified as either congenital or acquired. Patient history is critical in distinguishing congenital from acquired AVM [1]. A history of molar disease and multiple dilation and curettage, as seen in our patient, predisposes a patient to acquired AVM. AVM usually develop slowly over a long period before becoming symptomatic; however, rapid growth may occur in response to a hormonal influence such as during pregnancy or trauma [5]. The dilation of vessels during pregnancy as a consequence of vasoactive hormones is well known [6]. As a result of vasoactive hormonal influence during pregnancy, AVMs also expand due to uterine stretching and enlargement. In the years preceding this case, our patient had multiple pelvic MRI and ultrasound evaluations as well as two uterine arterial embolization with no decrease in AVM lesions. There was almost no adequate vessels-free area along the anterior aspect of uterus. Additionally, there were intermittent periods of vaginal bleeding during this pregnancy possibly due to rapid extension of a lesion which had been identified by repeated color Doppler ultrasonography.

Classical treatment for uterine AVMs was uterine artery ligation and hysterectomy [3]. It was not a good option, however, in our case considering the existence of extensive pelvic AVMs. Owing to the presence of innumerable intra and extra uterine microscopic shunts, hys- terectomy would be ineffective-potentially leading to a catastrophic life-threating hemorrhage [6]. In recent years, transcatheter uterine arterial embolization has been implicated as an alternative treatment option [6]. We understand uterine artery embolization should be performed before surgical treatment of nonmalignant pelvic bleeding. However due to our patient's poor response to previous unsuccessful uterine artery embolization, we questioned whether one more uterine arterial embolization would be sufficient to prevent major bleeding from this large AVM lesion. The high index of suspicion for a life threatening hemorrhage discouraged us from only relying on transcatheter uterine arterial embolization during the C-section.

The surgical plan consisted of a two-tiered approach. The initial plan (plan A) consisted of a preoperative transcatheter balloon-assisted occlusion to the aortic and right iliac artery followed by delivering the baby via Csection and post-operative transcatheter uterine arterial embolization. If there was massive hemorrhage intraoperatively and balloon inflation was unsuccessful then the patient would undergo a hysterectomy and/or an emergency ECLS would be instituted (plan B). ECLS has been suggested as a therapeutic option in refractory cardiac arrest, multiple trauma, surgery bleeding, cardiogenic shock, witnessed cardiac arrest, massive pulmonary embolism, near drowning, drug overdose, and severe hypothermia [7-9]. The advantage of ECLS was using the femoral-femoral vascular cannulation approach for cardiopulmonary bypass would be to avoid the complications associated with sternotomy. Encouraging results from this technique have been published recently by several teams where good neurological outcome has been observed in up to $20 \%$ to $30 \%$ of cases [9].

A cautious anesthesia strategy was implemented as well. To minimize the exposure and potentially negative impact of volatile anesthetics exposure to the fetus we utilized mild sedation instead of general anesthesia during placement of the balloon artery occlusion catheters. Fetal compromise secondary to extended periods of general anesthesia has been reported. Some authors observed that there was increased incidence of low 1 minute Apgar scores among infants exposed to general anesthesia for elective C-section. Additionally, infants exposed to general anesthesia are more likely to be depressed and require active resuscitation [10]. Interestingly, contrary to previous research findings, a new study demonstrated that the combination of nitrous oxide and isoflurane induced a significant change in gene expression and cell death of neuronal tissues in post-natal rats [11]. According to Danforth's Obstetrics and Gynecology, the prolonged neonatal effects have led to the virtual elimination of the use of general anesthesia during labor and birth 
[12]. Sedation with small dose of midazolam during the third trimester has no negative effect on fetus is well known; therefore mild sedation under management anesthesia care during insertion of balloon-assisted occlusion catheters in our case was an appropriate choice. We were not able to avoid the use of general anesthesia since there was a high possibility of the parturient requiring a hysterectomy and massive resuscitation during $\mathrm{C}$-section delivery.

It is our strong belief that although we did not face a major disaster during this $\mathrm{C}$-section, our comprehensive plan and multi-disciplinary approach were the key in carrying out a successful patient management plan with a significant potential for life-threatening hemorrhage.

\section{REFERENCES}

[1] R. Bagga, P. Verma, N. Aggarwal, V. Suri, R. J. Bapuraj and N. Kalra, "Failed Angiographic Embolization in Uterine Arteriovenous Malformation: A Case Report and Review of the Literature," The Medscape Journal of Medicine, Vol. 10, No. 1, 2008, p. 12.

[2] H. M. Fraser, "Clinical Implications of Disturbances of Uterine Vascular Morphology and Function," Best Practice \& Research Clinical Obstetrics \& Gynaecology, Vol. 14, No. 6, 2000, pp. 937-951.

[3] T. K. Ghosh, "Arteriovenous Malformation of the Uterus and Pelvis," Obstetrics \& Gynecology, Vol. 68, No. 3, 1986, pp. 40S-43S.

[4] P. O'Brien, A. Neyastani, A. R. Buckley, S. D. Chang and G. M. Legiehn, "Uterine Arteriovenous Malformations: From Diagnosis to Treatment," Journal of Ultrasound in Medicine, Vol. 25, No. 11, 2006, pp. 1387-1392,
1394-1395.

[5] K. D. Calligaro, T. V. Sedlacek, R. P. Savarese, P. Carneval and D. A. DeLaurentis, "Congenital Pelvic Arteriovenous Malformations: Long-Term Follow-Up in Two Cases and a Review of the Literature," Journal of Vascular Surgery, Vol. 16, No. 1, 1992, pp. 100-108. doi:10.1016/0741-5214(92)90425-8

[6] G Zanetta, G. Rangoni, M. Zanetta and G. Corsi, "Conservatively Treated Pelvic Arteriovenous Malformation: Noninvasive Sonographic Monitoring during Subsequent Pregnanc," Journal of Clinical Ultrasound, Vol. 25, No. 7, 1997, pp. 401-404.

doi:10.1002/(SICI)1097-0096(199709)25:7<401::AID-JC U10>3.0.CO;2-I

[7] G. Gravlee, R. F. Davis, A. H. Stammers and R. M. Ungerleider "Cardiopulmonary Bypass: Principles and Practice," Lippincott Williams \& Wilkins, Philadephia, 2008.

[8] A. M. Philipp, et al., "Extracorporeal Membrane Oxygenation in Severe Trauma Patients with Bleeding Shock," Resuscitation, Vol. 81, No. 7, 2010, pp. 804-809. doi:10.1016/i.resuscitation.2010.02.020

[9] M. Le Guen, et al., "Extracorporeal Life Support Following Out-of-Hospital Refractory Cardiac Arrest," Critical Care, Vol. 15, No. 1, 201, p. R29. doi:10.1186/cc9976

[10] D. H. Chestnut. "Obstetric Anesthesia: Principles and Practice," 3 rd Edition, Mosby, Philadelphia, 2004.

[11] F. Liu, et al., "Inhalation Anesthesia-Induced Neuronal Damage and Gene Expression Changes in Developing Rat Brain.” Systems Pharmacology, Vol. 1, No. 1, 2012, pp. 1-9. doi:10.2478/sph-2012-0001

[12] J. R. Scott, et al., "Danforth's Obstetrics and Gynecology," 9th Edition, Lippincott Williams \& Wilkins, Philadephia, 2003. 